\title{
Herbicide Absorption and Translocation in Plants using Radioisotopes
}

\author{
Vijay K. Nandula and William K. Vencill*
}

2,4-D, discovered independently in the United States and Europe in the mid-1940s, was one of the first synthetic herbicides to be used selectively for weed control (Cobb and Reade 2010). Since then, several herbicides belonging to different chemical classes and possessing diverse mechanisms of action have been synthesized and marketed globally. Herbicides have vastly contributed to increasing world food, fiber, fuel, and feed production in an efficient, economic, and environmentally sustainable manner. Before receiving regulatory approval, all herbicides (pesticides) undergo rigorous testing for their toxicological, residual, physicochemical, and biological properties. Additionally, herbicides are suitably formulated to reach their target site and maximize their efficacy on target weeds while being safe on crops. One of the main principles behind the design of herbicide formulations is getting the active ingredient across the leaf surface barrier in the case of POST herbicides or into the root with regard to soil-applied herbicides (PRE and PPI). As a part of these tests, the absorption and translocation of herbicides is routinely investigated using various analytical techniques and complementary approaches to detect localization in plant tissues and organelles. Since some herbicide resistance mechanisms involve altered absorption and translocation of herbicides (i.e., glyphosate and paraquat), this is an important component in the study of herbicide resistance mechanisms as well. Radioactive herbicides have been in use for over half a century in the study of herbicide absorption and translocation in plants. An up-to-date, organized description and standardization of research procedures and methodology on the use of radioisotopes in the study of herbicide absorption and translocation in plants is lacking in the literature. This article attempts to fill this void.

DOI: 10.1614/WS-D-13-00107.1

* Research Plant Physiologist, Crop Production Systems Research Unit, Agricultural Research Service, United States Department of Agriculture, Stoneville, MS 38776; Professor, Department of Crop and Soil Sciences, The University of Georgia, Athens, GA 30602. Corresponding author's E-mail: vijay.nandula@ars.usda.gov

\section{Terminology (Mostly Pertains to Radioactive Isotopes)}

Mass Balance. This item is described as the sum of the radioactivity measured in all plant parts, leaf (or root) washes, and root exudates and calculated as a proportion of applied radioactivity at the start of the experiment (Kniss et al. 2011). It is expressed as percent recovery. Mass balance will help account for losses through volatilization, radioactive dust, root exudation, and experimental techniques.

Uptake or Absorption. It is the amount or quantity or percentage of applied active ingredient that has been absorbed by the leaves or roots of the treated plant. This is also calculated as the sum of the radioactivity measured in all parts and is generally expressed as percentage of applied radioactivity.

Translocation or Movement. This is defined as the sum of radioactivity recovered in all plant parts except the treated leaf (or roots) and is generally expressed as percentage of absorbed radioactivity.

Distribution. This is the fractionation or division of accumulated radioactivity in predetermined separated parts of the treated plant including treated leaf (or root). It is recommended to be expressed as a percentage of absorbed radioactivity.

\section{Units of Measurement}

The commonly accepted unit of measurement of radioactivity is the becquerel $(\mathrm{Bq})$, derived from the International System of Units (Système international d'unités). It is defined as:

$$
1 \text { becquerel }(\mathrm{Bq})=1 \text { disintegration } \mathrm{s}^{-1}(\mathrm{dps})
$$

However, kilobecquerel $(\mathrm{kBq})$ is a more appropriate unit for research and laboratory samples of radiolabeled herbicides. Other common units and conversions used in measuring radioactivity are: 


$$
\begin{aligned}
& 1 \mathrm{~Bq}=60 \text { disintegrations } \min ^{-1}(\mathrm{dpm}) \\
& \begin{array}{l}
1 \mathrm{kBq}=5.946 \times 10^{4} \mathrm{dpm} \\
1 \text { microcurie }(\mu \mathrm{Ci})=37 \mathrm{kBq} \\
=2.2 \times 10^{6} \mathrm{dpm}=3.7 \times 10^{4} \mathrm{dps} \\
1 \text { millicurie }(\mathrm{mCi})=37 \text { megabecquerels }(\mathrm{MBq}) \\
=3.7 \times 10^{4} \mathrm{kBq}
\end{array}
\end{aligned}
$$

\section{Regulatory Requirement}

This section has mostly been adapted from a U.S. Environmental Protection Agency website (http://www.epa.gov/radtown/research-lab.html). Researchers outside the United States should comply with locally relevant usage and documentation requirements. Radioactive materials are used in research studies to aid in the development of new products or understanding scientific concepts. Radioactive isotopes are either contained in miniature vials or bulky devices, for example, an X-ray machine. Radioactive materials have several uses in research: in the development of new cancer treatments in medicine, in enhancement of plant and animal production and protection in agriculture, in tracking pollutants in water and soil in environmental research, in the determination of age of rock formations and study of continental drift in geological research, and in archaeology for the determination of the age of artifacts using carbon $\left({ }^{14} \mathrm{C}\right)$ dating. Safety of the user and the general public is of utmost importance in the storage and application of radionuclides. A research facility must provide documented proof that it is capable of ordering, storing, and utilizing radioactive substances. Personnel using radioactive isotopes must be properly trained in the properties of the materials, personal protective equipment, and procedures for the appropriate use in research. Proper records of radioisotope use and disposal should be maintained at all times, which is essential to first responders during emergency situations. Air emission records from research facilities using radioisotopes should be maintained annually. All radioactive waste generated must be disposed of following local and federal regulations. The U.S. Nuclear Regulatory Commission is the main agency of the U.S. federal government that regulates medical, industrial, and academic uses of nuclear materials through a combination of regulatory requirements; licensing; safety oversight, including inspections and enforcement; operational experience evaluation; and regulatory support activities.

\section{Radioisotope Application Environment}

Herbicide absorption studies using radioisotopes are usually conducted in a greenhouse or controlled environment chamber (growth chamber) to reduce variability and improve the reproducibility of the experiment. When using a greenhouse in temperate environments, we recommend performing (radioactive) herbicide absorption studies during the spring (February to April) or fall (September to November) months when the outside temperatures are not prohibitively high and light intensity is moderate. Some researchers may prefer to treat plants in a growth chamber and leave them there until the conclusion of the experiment. Under such situations, it is advisable to let experimental plants acclimatize to these growing conditions for at least $24 \mathrm{~h}$ before application of cold (nonradioactive) and hot (radioactive) herbicide treatment. It is critical to repeat experiments under similar conditions as much as possible.

Before beginning any herbicide absorption and translocation experiment, the researcher should define the hypothesis being tested and carefully lay out the experimental details. Usually the researcher wants to examine the behavior of a new herbicide in a species of interest, or compare two or more herbicides, specific formulations, additives, or the effect of specific environmental parameters on herbicide behavior in plants. In recent years, many researchers have conducted herbicide absorption and translocation studies to elucidate herbicide resistance mechanisms in weeds such as horseweed [Conyza canadensis (L.) Cronq.] (Koger and Reddy 2005), Italian ryegrass [Lolium perenne L. ssp. multiflorum (Lam.) Husnot] (Nandula et al. 2008), and rigid ryegrass [Lolium rigidum Gaud] (Lorraine-Colwill et al. 2003). Radioactive herbicides are expensive and difficult to dispose of so they should only be used with clear research objectives in mind.

The following procedures outline practices to conduct experiments on herbicide absorption from a foliar application, application of herbicide solution to the treated leaf, root absorption experiments, measurement of unabsorbed herbicide, and herbicide translocation from the treated plant. Autoradiography and phosphorimaging techniques will be outlined. Finally, some nonradiolabeled techniques 
for measuring absorption and translocation of herbicides in plants are discussed.

\section{Absorption}

Despite the frequent implementation of foliar herbicide absorption studies, a prescribed protocol for conducting such research is lacking in the literature. Perhaps the source that comes closest in meeting this need is a manual titled "Research Methods in Weed Science" (Camper 1986). This reference is outdated and does not meet today's requirements. For example, newer techniques such as phosphorimaging, more robust statistical models for data analysis, and more stringent procedures in the use and disposal of radioisotopes are commonplace now. Additionally, several nonradioactive bioassays, described in detail in other articles in this issue, supplement a researcher's tool kit in the study of herbicide absorption and translocation in plants. In an effort to fill this gap, Kniss et al. (2011) published an elegant review that provides a framework of procedures for conducting herbicide absorption studies and analysis of related data. Herbicide absorption data from 27 published manuscripts between 2006 and 2010 were analyzed. The reader is advised to consult this report for further details on regression analysis and modeling of maximum herbicide absorption and absorption rate data. Researchers are strongly encouraged to conduct a thorough literature review on the herbicide being investigated to avoid unnecessary repetition of research studies that may generate data similar to what may already be available.

\section{Translocation}

Translocation is an important attribute to consider when dealing with systemic herbicides such as glyphosate, acetolactate synthase inhibitors, and graminicides. It has gained further prominence since its implication as a mechanism of resistance to glyphosate in several weeds (Dinelli et al. 2008; Feng et al. 2004; Koger and Reddy 2005; Nandula et al. 2008; Perez-Jones et al. 2007; Wakelin et al. 2004). Overspray with nonradioactive glyphosate should be carefully considered when studying glyphosate translocation. For example, a narrow difference in translocation between resistant and susceptible weed populations may be disguised by a self-limiting herbicide such as glyphosate (further movement of herbicide in a plant to sinks is restricted because of saturation of sinks and establishment of a reverse concentration gradient from sink to source leaves) when applied at a labeled rate (Kniss et al. 2011; Shaner 2009). Conversely, overspray with glyphosate is recommended when differences between resistant and susceptible weed biotypes are expected to be significant (Shaner 2009). This information can be gleaned from preliminary experiments such as dose-response assays (to determine the resistance factor/index) or a test trial with and without overspray at a sublethal rate of $1 / 4 \times$ or $1 / 2 \times$ of the labeled rate. Translocation studies usually follow radioactive herbicide absorption studies, but are more time consuming and labor intensive. Although the study of absorption pattern within hours after herbicide application is important, translocation can occur several hours to several days after herbicide application. Hence, time points for measuring translocation should be carefully considered on the basis of published research as well as initial experiments. The most commonly used procedure for measuring translocation is by biological oxidation, where dried radioactivity-treated plant samples are burned in the presence of $\mathrm{O}_{2}$ and the resulting ${ }^{14} \mathrm{CO}_{2}$ is captured in a special solvent and radioactivity is measured by liquid scintillation counting.

\section{Preparation (Partially Adapted from Kniss et al. [2011])}

Step 1. Select uniform plants of the same age or growth stage as much as possible.

Absorption (Abs) Tip \#1. The age/size of treated plants must be as close to the growth stage at which labeled rates of the herbicide are applied.

Step 2. Use at least six time points in addition to a 0 time point of tissue harvest (Figure 1); under conditions of limited resources, it is better to increase the number of time points and reduce the number of replications. Include nontreated plants as a blank or control.

Note. Although this is a good starting point for balancing number of time points vs. number of replications per time point, fewer time points that are statistically robust could be better than more time points that have large standard deviations. Such information can be gleaned from the first run of the experiment. Conduct preliminary studies, where information is lacking in the literature, to 


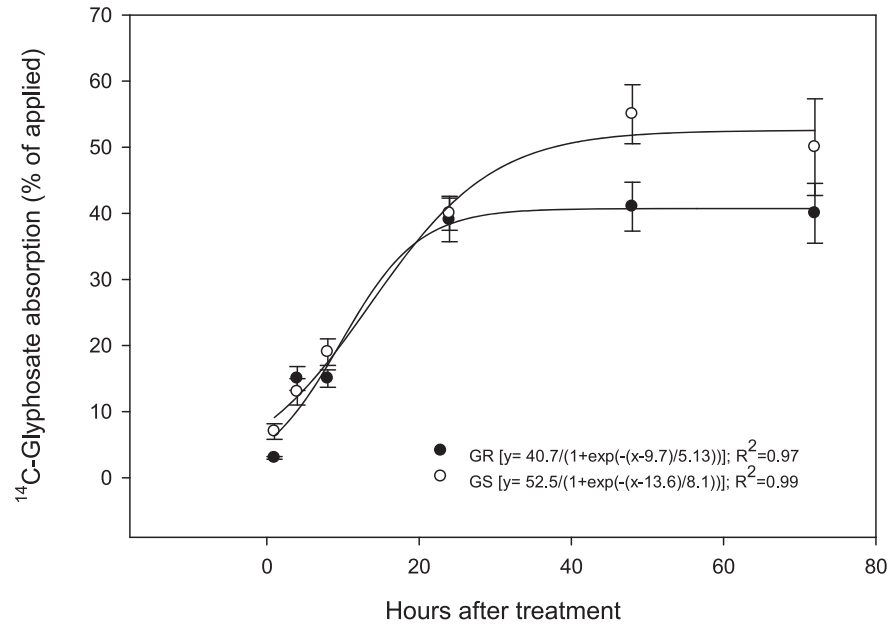

Figure 1. Absorption pattern of ${ }^{14} \mathrm{C}$-glyphosate in glyphosateresistant (GR) and -susceptible (GS) tall waterhemp (Amaranthus tuberculatus) populations. Vertical bars represent standard error of mean. From Nandula et al. (2013). Note the six time points.

determine optimal time points. The time course for absorption and mobility of any herbicide in the plant varies by weed species, and perhaps even by population, biotype, or ecotype. We cannot assume that optimum time point is the same for one herbicide across species. Where time course of absorption of a herbicide is already known, this experiment could be redundant. Also, available information (or lack thereof) on translocation of the herbicide will determine the design of the absorption studies.

Step 3. Application of Radioactive Treatment Solution. On the day before initiating the experiment, label plants that are to be treated. Cover the leaf or leaves predetermined for radioisotope treatment with aluminum foil or polythene material (Saran ${ }^{\circledR}$ wrap, corner of Ziplock ${ }^{\circledR} /$ Whirl-Pak ${ }^{\circledR}$ bags, etc.) or paper (coin envelopes or soil sampling bags) (Figure 2).

Step 4. Overspray the plants with unlabeled or "cold" (without radioisotope) herbicide solution containing the herbicide at full labeled rate (sublethal rates may be used in preliminary experiments or under conditions where an accession of a weed species is extremely sensitive to a herbicide at full rate), label-suggested adjuvant(s), and water, followed by immediate removal of aluminum foil or polythene material.

Abs Tip \#2. Overspray with cold herbicide is done to mimic real-world conditions and to not cause artificially high translocation due to high concentration in one plant part.
Note. In general, full labeled field rates for herbicide applications are used as standard. Sublethal rates may be applicable in certain situations, but do not provide the same physiological response as a full rate. An alternative could be using a herbicide rate that causes a $50 \%$ reduction in plant growth, especially, in the case of tolerant weed species. This can be obtained by conducting a dose-response study before conducting an absorption/translocation experiment.

Step 5. Radioactive Treatment Solution. Prepare a solution containing the formulated herbicide (at a concentration equivalent to the amount of herbicide active ingredient at predetermined rate dissolved in preset volume of water recommended for field application; for example, glyphosate at $0.84 \mathrm{~kg} \mathrm{ae} \mathrm{ha}^{-1}$ in $140 \mathrm{~L}$ of water), recommended adjuvants (including surfactants and conditioning agents), radiolabeled herbicide, and distilled water. This preparation could be made using an aliquot of the overspray cold herbicide solution and then supplementing the solution with the calculated radioactive herbicide or newly made (except when measuring very small quantities of one or more ingredients of the solution becomes prohibitive). Check the actual radioactivity in the prepared solution, as a safeguard against potential error in calculations or mixing, before applying on plants.

Note. Distilled water will help avoid changes in $\mathrm{pH}$ of the treatment solution (both overspray and radioactivity), which may adversely affect absorption of certain herbicides. Also, the presence of divalent cations such as $\mathrm{Mg}^{2+}$ and $\mathrm{Ca}^{2+}$ can limit uptake of herbicides such as glyphosate by forming irreversible chemical complexes in the treatment solution.

Step 6. Double-check calculations to make sure that the amount of radiolabeled herbicide added to the treatment solution does not alter the herbicide concentration.

Step 7. Within $30 \mathrm{~min}$ to $1 \mathrm{~h}$ after overspray, apply radioactive isotope of herbicide prepared as described above. The amount of radioactivity to be applied per plant depends mostly on the specific activity of radioactive herbicide stock solution and to a lesser extent on the radiochemical purity. The rule of thumb is to apply high enough radioisotope to ensure that recovered samples have sufficient radioactivity to provide a reliable count. For absorption only (without any follow-up translocation measurement), no less than $170 \mathrm{~Bq}$ per plant should be applied for most annual weeds. 


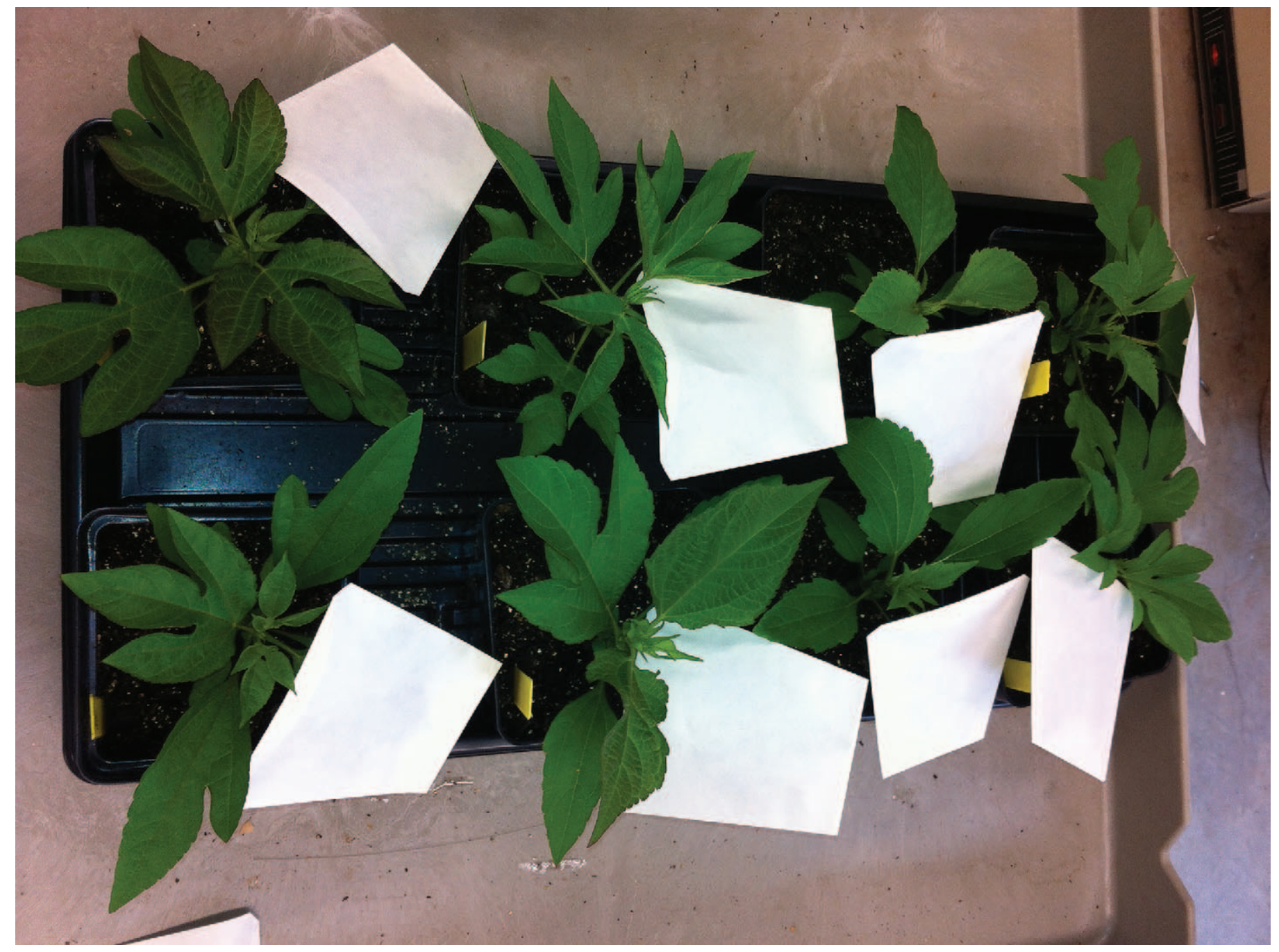

Figure 2. Illustration of covering of leaf (to be treated with radioactive herbicide solution) at time of overspray with cold herbicide.

A commercial source for radiolabeled herbicides is American Radiolabeled Chemicals, Inc. (St. Louis, MO 63146). Agrochemical companies are another resource for radioactive herbicide samples. Typically, a volume of 5 to $10 \mu \mathrm{l}$ of the treatment solution is applied to the adaxial (dorsal or upper) leaf surface with a microapplicator. The treatment solution is commonly applied in the form of tiny droplets mimicking a spray deposit or smeared on the leaf surface on either side of the midrib. The microapplicator can be a micropipet or a microsyringe.

Abs Tip \#3. We recommend a microsyringe (Hamilton ${ }^{\circledR}$ brand 1800 series gas-tight syringes with fixed needle, 26 s gauge, type 2 needle, 10 - to 500- $\mu$ l range, Hamilton Company, Reno, NV 89520). This syringe needle is blunt-edged and will not rupture or scar the leaf epidermis (Figure 3).
Abs Tip \#3. Use of Lanolin. Leaf surfaces of certain weed species are highly hydrophobic due to high wax content on the leaf surface, which could pose a challenge for herbicide penetration. It is recommended to apply radioactive herbicide treatment solution within a lanolin (product \# L7387, Sigma-Aldrich Co., LLC, Saint Louis, MO 63103) ring. A lanolin ring of any desired diameter (depending on area of leaf lamina or blade) could be achieved using a blunt accessory such as a cotton swab or a glass rod. Wipe off the lanolin after the tissues have been harvested.

Note. It is very likely that certain lipophylic (fat loving/soluble) herbicide molecules such as atrazine, carfentrazone, fluazifop, oxyfluorfen, tralkoxydim, etc. may separate out on contact with lanolin. Small-scale preliminary studies are recommended before designing full-scale studies toward investi- 


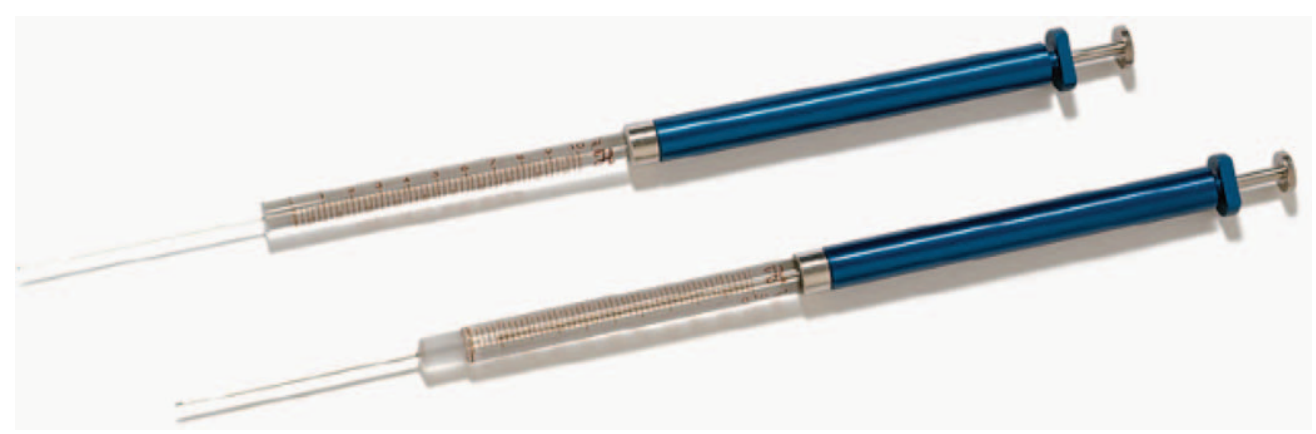

Figure 3. Recommended blunt-edged syringe for application of radioactive herbicide treatment solution (with permission from Hamilton Company, Reno, NV 89502).

gating absorption patterns of lipophylic herbicides using the lanolin ring technique. Examples of hydrophilic herbicides include glyphosate, paraquat, and sulfonylureas.

Translocation (Trans) Tip \#1. Save an aliquot of the root rinsate for measurement of radioactivity to detect for herbicide exudation. In the case of root absorption studies (described in a later section) an aliquot of the hydroponic solution or sand rinsate should be set aside.

Step 8. Determine the mass balance of applied radioactive herbicide. A mass balance of $80 \%$ or more should be targeted to account for volatilization losses, radioactive dust, root exudation, and differences in experimental technique of researchers/ personnel. Mass balance can also be referred to as "percentage of recovery," which is the ratio of sum of radioactivity via biological oxidation and leaf/ root wash (unabsorbed herbicide) to total radioactivity applied to an individual plant.

Note. It is essential for all researchers using radioactive isotopes, new or experienced alike, to determine mass balance of radioactivity over the course of an experiment. If there is substantial difference in the percentage of recovery of radioactivity at the beginning of a study compared with the middle or a later stage, crucial information on the underlying interactions between the applied herbicide and treated plant surface may be lost and conclusions drawn could be misrepresentation of what is actually happening in the plant, physiologically.

Step 9. Measurement of Absorbed Herbicide. At each harvest time, remove the treated leaf (leaf blade in grasses) and immerse in 10 to $20 \mathrm{ml}$ of distilled water or an aqueous solution (10 to $50 \%$ ) of an organic solvent such as methanol.

Abs Tip \#4. The choice of distilled water or the appropriate organic solvent is based on previously published research or the herbicide manufacturer's recommendations for removal of unabsorbed (radioactive) herbicide.

Step 10. Before harvest, measure the chosen solvent into 20 -ml glass scintillation vials (catalog \# 03337-7, Thermo Fisher Scientific Co., Pittsburgh, PA 15205).

Step 11. After treated leaf immersion, shake capped vials gently for 10 to $20 \mathrm{~s}$ to remove nonabsorbed radioactive herbicide remaining on the leaf surface.

Step 12. Remove the leaf from first wash and repeat the leaf wash with an additional equal volume of the solvent, measured into a separate vial.

Step 13. Mix two 1-ml aliquots of each leaf wash with $10 \mathrm{ml}$ of scintillation cocktail $\left(\right.$ Ecolume ${ }^{\circledR}$, ICN, Costa Mesa, CA 92626). Similarly, two aliquots of the nutrient solution in the case of hydroponic root absorption studies may be measured for radioactivity.

Step 14. Biological Oxidation and Liquid Scintillation Counting. Harvest plants at predetermined harvest times. After the treated leaf or blade (in grasses) is excised, wash to remove nonabsorbed herbicide, and set aside, divide rest of the plant into shoot above treated leaf (SATL), shoot below treated leaf (SBTL), and roots. 
Note. SATL includes the apical and axial (lateral) growing points. The SBTL includes the part of the shoot from below the treated leaf up to the soil surface. It may include some lateral meristems.

Note. If the amount of material exceeds what can be oxidized, then a composite sample and subsamples can be made (e.g., Camacho and Moshier 1991).

Step 15. Wash roots in minimal amount of water to remove soil medium.

Step 16. Wrap the respective plant parts in a single layer of paper (Kimwipes ${ }^{\circledR}$ 34155, Kimberly-Clark Corporation, Roswell, GA 30076), place in a glass scintillation vial, and oven dry at $60 \mathrm{C}$ for $48 \mathrm{~h}$.

Step 17. Weigh dried tissue samples along with paper. Obtain average weight of 10 papers and subtract from weights of samples plus paper to obtain tissue sample weights. These weights are used to calculate herbicide concentration values.

Step 18. Combust oven-dried plant samples in a biological oxidizer (Sample Oxidizer Model 307, PerkinElmer, Waltham, MA 02451) and the evolved ${ }^{14} \mathrm{CO}_{2}$ is trapped in $10 \mathrm{ml}$ of Carbosorb E (PerkinElmer, Meridian, CT 06450) and $10 \mathrm{ml}$ Permaflour E+ (PerkinElmer), or for the Harvey OX500, OX-160 ${ }^{14} \mathrm{C}$-cocktail.

Note. Biological oxidizers are also available from other manufacturers such as R. J. Harvey Instrument Corp., Tappan, NY 10983.

Trans Tip \#2. Before oxidation, it is advisable to dry samples for 15 to $30 \mathrm{~min}$ at $60 \mathrm{C}$ to expel any traces of moisture.

Step 19. Quantify radioactivity from oxidations using liquid scintillation spectrometry (e.g., TriCarb 2500 TR liquid scintillation analyzer, PerkinElmer, or LS 6500, Beckman-Coulter, Inc. (Brea, CA 92821).

Trans Tip \#3. Total of radioactivity recovered in all plant parts except the treated leaf is designated as translocated ${ }^{14} \mathrm{C}$ and expressed as percentage of absorbed.
Step 20. Use regression analysis to describe herbicide absorption over time.

Note on Herbicide Metabolism. Detection of translocation or movement of a herbicide away from the treated leaf in a plant does not necessarily mean that the applied herbicide is remaining in its parent form. The radiolabeled herbicide could have been converted to a nonphytotoxic metabolite. Conceivably, movement of the metabolite could be restricted and may not be readily translocated. Potential for metabolism of a herbicide in a weed species should be investigated on the basis of previously reported research and other related information available in the literature. Radioactive labeling of a herbicide molecule and its implications in translocation measurement is further discussed in the Autoradiography section.

\section{Absorption via Roots}

With the highly effective weed control systems offered by glyphosate-resistant crops and the associated benign environmental properties of glyphosate, absorption studies with PPI, PRE, or POST herbicides with soil activity have become rare. However, with evolution of glyphosateresistant (GR) weeds, land managers are increasingly adding PPI and PRE herbicides to their weed management portfolios in agronomic crops. Hence, resurgence in research on herbicide absorption through the root system is likely.

Root absorption studies are commonly carried out in hydroponic growing conditions or a suitable soil medium such as sand (that minimizes adsorption of the herbicide to soil particles. The most popular nutrient solution for water culture studies is Hoagland's solution (Hoagland and Arnon 1950). The following procedure is commonly practiced:

Step 1. Prepare the radioactive herbicide treatment solution by mixing formulated herbicide product and a radioactive aliquot in distilled water to reach the desired concentration.

Step 2. Apply the radioactive treatment solution evenly to the soil (or liquid growing medium) through a micropipet. Alternatively, the herbicide treatment solution could be sprayed onto the soil medium with an indoor sprayer. However, this procedure is expensive and requires a large quantity of radioisotope and subsequent disposal to which most researchers will not have access. 
Note. In the case of a hydroponic system, the herbicide concentration in the treatment solution should be higher than desired so that after addition to the liquid medium the final concentration is at the desired levels. Prepare a stock solution of herbicide and use the dilution formula

$$
C 1 V 1=C 2 V 2
$$

where $C 1$ and $V 1$ are the stock concentration and volume, respectively; and $C 2$ and $V 2$ are the final concentration and volume of the hydroponic solution. Cover the containers holding the hydroponic solution with aluminum foil or black polythene/plastic/paper to prevent chemical changes to solution ingredients via oxidation and photolysis.

Step 3. For PPI or PRE, plant the seed on the soil medium (sand; sand is the best alternative to field soil or other commercial potting media) or anchor on a liquid medium. For POST treatments, germinated seedlings are transplanted into soil or inserted in suitable glass or plastic containers holding the nutrient solution.

Note. In hydroponic studies, the nutrient solution requires constant aeration for oxygenation and nutrient circulation to the roots. To avoid setup of an elaborate aeration system and potential contamination with radioactive material, we recommend gentle swirling or movement of plant containers several times a day during the course of the experiment.

Step 4. At predetermined harvest times, remove the treated plants and rinse the roots to remove excess nutrient solution and sand adhering to root surfaces.

\section{Autoradiography}

Autoradiography can be defined as the development of an image, on an X-ray film or a phosphorscreen coated with a specialized material such as barium fluorohalides, of the two-dimensional pattern of emission of beta $\left({ }^{14} \mathrm{C},{ }^{3} \mathrm{H}\right)$ or gamma $\left({ }^{32} \mathrm{P}\right)$ rays produced from decay of radioactive compounds. Almost all herbicides are organic chemicals (contain a carbon moiety) and have traditionally been tagged with ${ }^{14} \mathrm{C}$ atoms at selected locations on the parent molecule in their chemical structure for use in radiolabeled isotope studies. Labeling a side chain or a functional group may limit the use of the compound if it is rapidly metabolized or biologically cleaved from the main molecule. On the other hand, labeling a parent molecule will enable tracking of the herbicide using the techniques outlined in this article. Further, prior knowledge of potential metabolites of the herbicide as well as position of labeled atoms of the parent molecule will greatly assist a researcher. Herbicide movement in plants has been studied with the aid of X-ray film autoradiography for more than 50 yr. Whereas biological oxidation provides a quantitative estimate of bound herbicide in a treated plant, autoradiography offers a qualitative/relative measurement and localization of herbicide movement in the plant. Both have their respective applications in research on herbicide absorption and translocation in plants.

Procedure. Step1. Remove the treated leaf from radioactive herbicide-treated plants at predetermined harvest times and wash as described before to remove nonabsorbed radioactivity. Gently rinse roots with water to remove soil material and blot dry with paper towels.

Step 2. Mount plants on a 27 - by $21.25-\mathrm{cm}$ plain white paper. Evenly spread shoot and root parts and keep in place with the help of thin strips of clear office tape. Alternatively, plant parts could be mounted with paper glue. Press the mounted plant between one or more layers of newspaper and bookend with two hard cardboard sections. Hold the assembled plant press together with large binder clips and store at $-20 \mathrm{C}$ for later drying.

Autoradiography Tip \#1. Readjusting the position of the plant on the mounting paper could be a bit of a challenge when glue is sprayed on.

Note. Take care to avoid contact of the washed treated leaf with other parts of the plant. Treatment of plants (weed or crop) with ${ }^{14} \mathrm{C}$-labeled herbicide solution, harvest (including roots devoid of growing medium) at predetermined time intervals, and washing of treated leaf (leaves) to remove unabsorbed herbicide are achieved following procedures described in previous sections.

Step 3. Mount or adhere the plant sample(s) to a plain white background, avoiding contact of the treated leaf with other parts of the plant; press between one or more layers of newspaper; and bookend with two hard cardboard or metal surfaces, one on top and one on the bottom. 


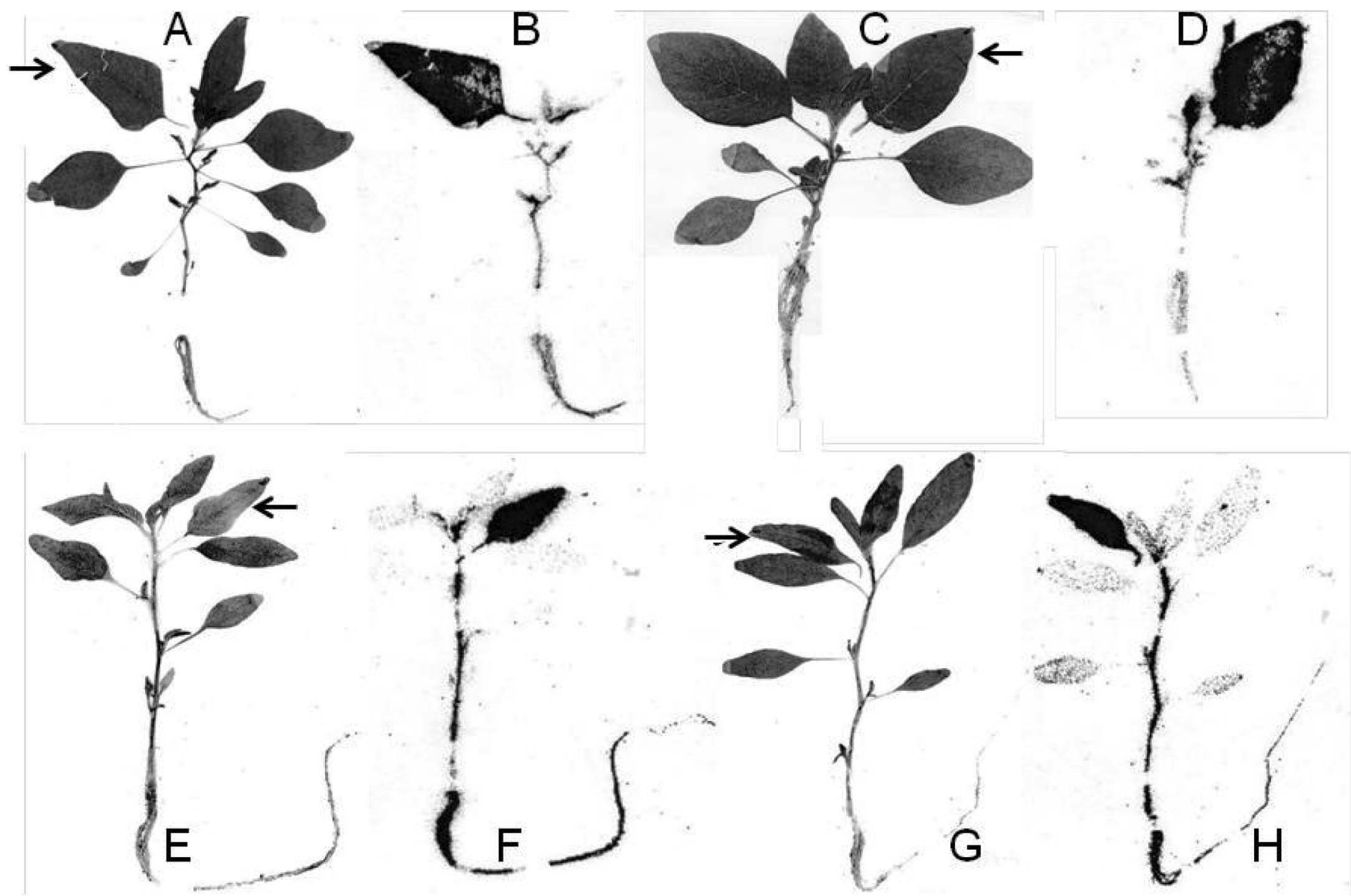

Figure 4. ${ }^{14}$ C-Glyphosate translocation in glyphosate-resistant ([GR], A to D) and -susceptible ([GS], E to H) tall waterhemp plants. Arrows indicate the treated leaf. First and third columns represent treated plants and second and fourth columns represent their corresponding phosphorimages (autoradiographs) at 24 and $48 \mathrm{~h}$ after treatment, respectively. From Nandula et al. (2013).

Step 4. Dry the plant press in a gravity convection oven at 40 to $60 \mathrm{C}$ for 24 to $48 \mathrm{~h}$.

Step 5. Expose the dried plants to X-ray film (multiple suppliers) for 4 wk.

Step 6. Develop and fix the exposed X-ray film according to manufacturer's instructions.

Note. Steps 4 and 5 require a darkroom. Also, the developing and fixing reagents require safe handling and disposal as hazardous chemicals. A more popular and safe, although expensive, approach for exposing the treated plant specimen to a film followed by image development is the use of phosphorimaging.

\section{Phosphorimaging}

This process involves exposure of plant specimens to a film containing a specialized coating for a period of $24 \mathrm{~h}$. A 1-d exposure time with phosphorimaging gave comparable or better results than a 3-wk exposure time with X-ray film (Wehtje et al. 2007). The film is then scanned by a laser, resulting in the development of an image, which is oftentimes $10 \times$ more sensitive compared with a X-ray autoradiograph. There are several manufacturers of phosphorimaging equipment, GE Healthcare Bio-Sciences Group, Perkin-Elmer, BioRad to name a few. We have had positive experience with the Typhoon FLA 7000 IP biomolecular imager from GE Healthcare. It could be upgraded or substituted for a more versatile version (Typhoon FLA 7000) that facilitates nonradioactive fluorescence and chemiluminescence measurements in addition to phosphorimaging.

Step 1. Follow steps 1 to 3 of autoradiography outlined before.

Step 2. Dry the plant samples in a gravity convection oven set at $60 \mathrm{C}$ for $24 \mathrm{~h}$.

Step 3. Cool the dried sample to room temperature, place the plant in a $20-$ by $40-\mathrm{cm}$ exposure cassette 


\section{Susceptible $\quad$ Tolerant $2 \quad$ Tolerant 1}

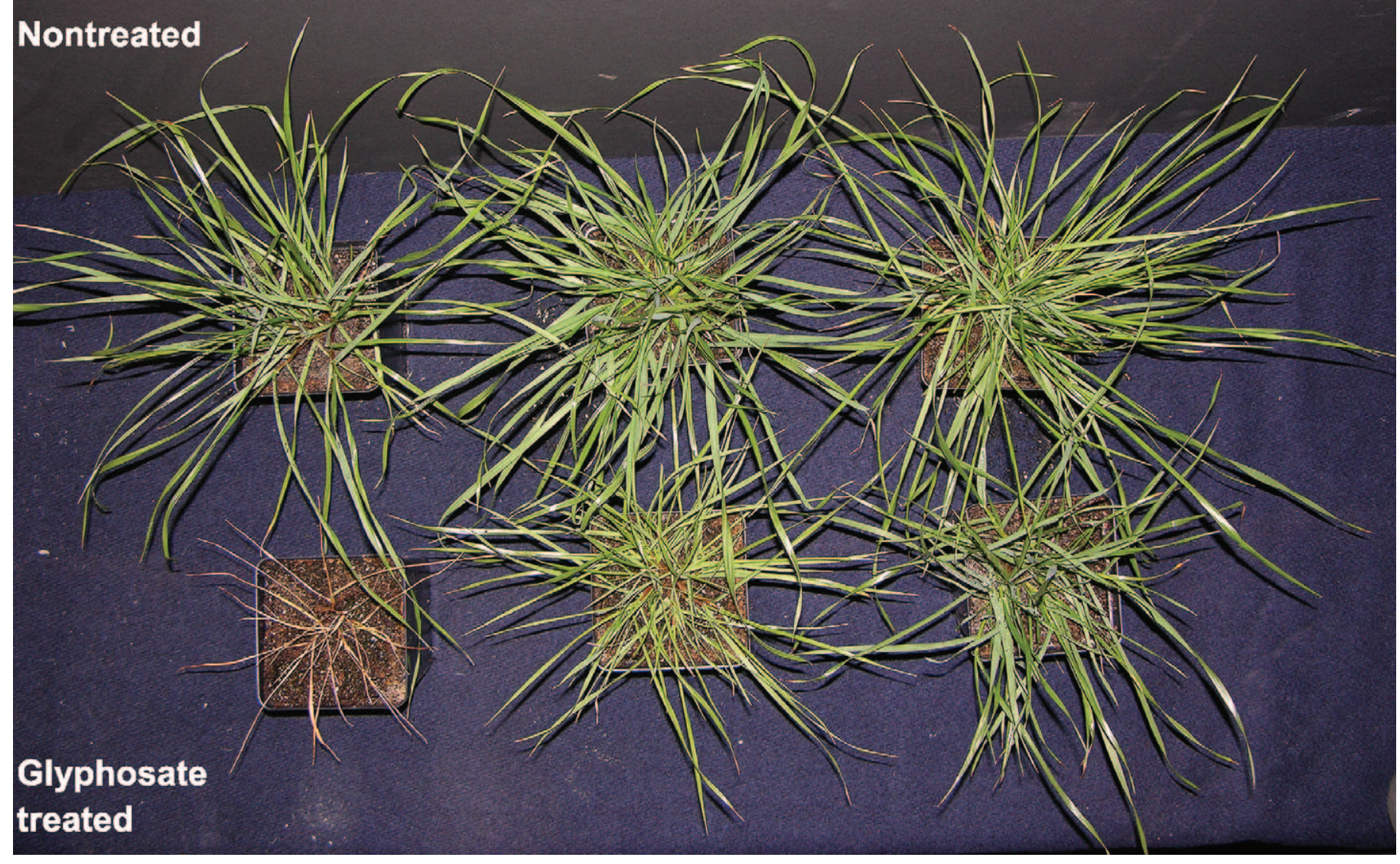

Figure 5. Glyphosate-susceptible S (left column), -tolerant T2 (middle column) and T1 (right column) Italian ryegrass populations from Mississippi. Upper and bottom rows represent nontreated and treated plants, respectively. A solution containing a commercial formulation of glyphosate (potassium salt) at a concentration of $0.84 \mathrm{~kg} \mathrm{ae} \mathrm{ha}^{-1}$ rate in $190 \mathrm{~L}$ of water was applied with a microsyringe to the adaxial surface of the third fully expanded leaf blade of $10-$ to $15-\mathrm{cm}$-tall (four leaves, two to three tillers) Italian ryegrass plants as $101-\mu l$-droplets. Three weeks after treatment, the $S$ plant was killed, and the T1 and T2 plants survived with some growth reduction from glyphosate. From Nandula et al. (2008).

(GE Healthcare Bio-Sciences Corp., Piscataway, NJ 08855), and bring into contact with a storage phosphor screen (BAS IP SR 2025 E, GE Healthcare Bio-Sciences Corp.) under low-intensity light.

Step 4. Place the plant-exposure cassette-screen assembly in a dark cabinet for $24 \mathrm{~h}$.

Step 5. Use a phosphorimager (Typhoon FLA 7000, GE Healthcare) to detect distribution of ${ }^{14} \mathrm{C}$ herbicide and develop an image (Figure 4).

\section{Nonradioactive Experimental Techniques}

Translocation: Single-Leaf Treatment with Nonradioactive Herbicide. There may be occasions when there is a research question to be answered and a radiolabeled herbicide is not available. As outlined below, there are ways to examine the translocation of a herbicide without a radiolabel tracer available. These techniques will work better with systemic than with nonsystemic herbicides.

Since their introduction in 1996, GR crops have been widely adopted by growers, especially in North and South America. Obvious consequences of the popularity of GR crops have been a drastic decrease in the diversity of herbicides applied to crop fields as well as a decline in the commercialization of new herbicides (old and new modes of action). A more costly outcome has been the evolution of resistance to glyphosate in several weed species (Heap 2013). A major research emphasis was placed by weed

Nandula and Vencill: Herbicide absorption and translocation • 149 
scientists over the past $15 \mathrm{yr}$ in understanding the mechanisms of glyphosate resistance in weeds, both for academic reasons and from a practical management standpoint. Reduced translocation, sometimes associated with reduced absorption, of glyphosate from treated source leaves to meristematic sinks in resistant plants compared with their susceptible counterparts was a relatively common phenomenon in some GR weed species. The reader is encouraged to consult other sources for more information on glyphosate resistance mechanisms. Since studies with radioactive isotopes can be expensive and labor intensive, we describe a simple experiment to investigate the role of translocation as a mechanism of glyphosate resistance.

Step 1. Prepare a solution containing glyphosate at $0.84 \mathrm{~kg}$ ae ha ${ }^{-1}(1 \times$ field rate) in $140 \mathrm{~L}$ (variable) of water. This solution is similar to glyphosate treatment solution applied as an overspray in studies with radioactive compounds or in testing glyphosate efficacy at labeled field rates.

Step 2. Place $10 \mu \mathrm{l}$ (in the form of 10 to 15 droplets) of glyphosate solution on the adaxial surface of the youngest fully expanded leaf or other chosen leaves with a micropipet.

Step 3. Harvest plants 2 to 4 wk after treatment, visually estimate percent control, and record aboveground shoot clipped and fresh weights (to calculate shoot fresh weight reduction). One of us and our colleagues have had good success using this technique (Koger and Reddy 2005; Nandula et al. 2008, 2012) (Figure 5).

This procedure could be extended to investigate translocation of other systemic herbicides. For example, the treated leaf can be excised at predetermined time points and the plants can be evaluated for the development of phytotoxic symptoms. Also, a study with a hydroponic system could be conducted in which the herbicide is added to the hydroponic solution, followed by removal of herbicide contact with plants by transfer of treated plants to a nonherbicide-containing solution.

\section{Statistical Analysis of Absorption and Translocation Data from Radioactivity Studies}

Herbicide absorption data can be analyzed following the procedures outlined by Kniss et al.
(2011). Nonlinear regression analysis may be applied to translocation data if the number of harvest intervals used is three or more. In case of head-to-head comparison of translocation between two or more accessions within a harvest time, data could be analyzed using common mean separation procedures. Autoradiography is purely a qualitative measurement, and hence does not involve any statistical application.

\section{Disclaimer}

Mention of trade names or commercial products in this publication is solely for the purpose of providing specific information and does not imply recommendation or endorsement by the U.S. Department of Agriculture.

\section{Literature Cited}

Camacho RF, Moshier LJ (1991) Absorption, translocation, and activity of CGA-136872, DPX-V9360, and glyphosate in rhizome johnsongrass (Sorghum halepense). Weed Sci 39: 354-357

Camper ND, ed. (1986). Research Methods in Weed Science. 3rd edn. Champaign, IL: Southern Weed Science Society. 486 p

Cobb AH, Reade JP (2010) Herbicides and Plant Physiology. 2nd edn. Oxford, UK: Wiley-Blackwell. 296 p

Dinelli G, Marotti I, Catizone P, Bonetti A, Urbano JM, Barnes J (2008) Physiological and molecular basis of glyphosate resistance in $C$. bonariensis (L.) Cronq. biotypes from Spain. Weed Res 48:257-265

Feng PC, Tran M, Chiu T, Sammons RD, Heck GR, CaJacob CA (2004) Investigation into GR horseweed (Conyza canadensis): retention, uptake, translocation and metabolism. Weed Sci 52:498-505

Heap IM (2013) International Survey of Herbicide Resistant Weeds. www.weedscience.org. Accessed July 9, 2013

Hoagland DR, Arnon DI (1950) The water-culture method for growing plants without soil. Circ. 347. Berkley, CA: University of California Agricultural Experiment Station

Kniss AR, Vassios JD, Nissen SJ, Ritz C (2011) Nonlinear regression analysis of herbicide absorption studies. Weed Sci 59:601-610

Koger CH, Reddy KN (2005) Role of absorption and translocation in the mechanism of glyphosate resistance in horseweed (Conyza canadensis). Weed Sci 53:84-89

Lorraine-Colwill DF, Powles SB, Hawkes TR, Hollinshead PH, Warner SAJ, Preston C (2003) Investigations into the mechanism of glyphosate resistance in Lolium rigidum, Pestic Biochem Physiol 74:62-72

Nandula VK, Ray JD, Ribeiro DN, Pan Z, Reddy KN (2013) Glyphosate resistance in tall waterhemp (Amaranthus tuberculatus) from Mississippi is due to both altered target site and non-target site mechanisms. Weed Sci 61:374-383

Nandula VK, Reddy KN, Koger CH, Poston DH, Rimando AM, Duke SO, Bond JA, Ribeiro DN (2012) Multiple resistance to glyphosate and pyrithiobac in Palmer amaranth (Amaranthus palmeri) from Mississippi and response to flumiclorac. Weed Sci 60:179-188 
Nandula VK, Reddy KN, Poston DH, Rimando AM, Duke SO (2008) Glyphosate-tolerance mechanisms in Italian ryegrass (Lolium multiflorum) from Mississippi. Weed Sci 56: 344-349

Perez-Jones A, Park K-W, Polge N, Colquhoun J, MallorySmith CA (2007) Investigating the mechanisms of glyphosate resistance in Lolium multiflorum. Planta 226: 395-404

Shaner DL (2009) Role of translocation as a mechanism of resistance to glyphosate. Weed Sci 57:118-123
Wakelin AM, Lorraine-Colwill DF, Preston C (2004) Glyphosate resistance in four different populations of Lolium rigidum as associated with reduced translocation of glyphosate to meristematic zones. Weed Res 44:453-459

Wehtje G, Miller ME, Grey TL, Brawner WR Jr (2007) Comparisons between X-ray film- and phosphorescence imaging-based autoradiography for the visualization of herbicide translocation. Weed Technol 21:1109-1114

Received July 18, 2013, and approved June 17, 2014. 